Review Article

\title{
Methods of Calculating Ionization Energies of Multielectron (Five or More) Isoelectronic Atomic Ions
}

\author{
Peter F. Lang and Barry C. Smith \\ Birkbeck College, University of London, School of Sciences, Malet Street, London WC1E 7HX, UK \\ Correspondence should be addressed to Peter F. Lang; p.f.lang@gmail.com
}

Received 21 March 2013; Accepted 18 April 2013

Academic Editors: A. Avramopoulos and Y. Luo

Copyright (C) 2013 P. F. Lang and B. C. Smith. This is an open access article distributed under the Creative Commons Attribution License, which permits unrestricted use, distribution, and reproduction in any medium, provided the original work is properly cited.

\begin{abstract}
We have previously used simple empirical equations to reproduce the literature values of the ionization energies of isoelectronic sequences of up to four electrons which gave very good agreement. We reproduce here a kinetic energy expression with corrections for relativity and Lamb shift effects which give excellent agreement with the literature values. These equations become more complex as the number of electrons in the system increases. Alternative simple quadratic expressions for calculating ionization energies of multielectron ions are discussed. A set of coefficients when substituted into a simple expression produces very good agreement with the literature values. Our work shows that Slater's rules are not appropriate for predicting trends or screening constants. This work provides very strong evidence that ionization energies are not functions of complete squares, and when calculating ionization energies electron transition/relaxation has to be taken into account. We demonstrate clearly that for particular isoelectronic sequences, the ionizing electrons may occupy different orbitals and in such cases more than one set of constants are needed to calculate the ionization energies.
\end{abstract}

\section{Introduction}

We have previously proposed a simple empirical equation to reproduce the literature values of the ionization energies of one-electron [1] and two-electron [2] atomic ions with very good agreement. This was recently extended to calculate ionization energies and first electron affinities of three and four electron ions [3] which also gave very good agreement with the literature values. However, we used a potential energy approach in our equation which has no theoretical basis.

With the development of quantum theory, the twoparticle problem can be solved exactly, and the kinetic energy of the electron in a hydrogen atom or hydrogen-like ion can be calculated using the Schrödinger equation. In 1930, Dirac [4] produced an equation which included a relativistic correction for the electron energy levels. Lamb and Retherford [57] were able to show that there is a small shift (now known as the Lamb shift) in the energy levels of the hydrogen atom not included in the Dirac equation. When the Lamb shift is taken into account, the actual ionization energies of one electron atoms are slightly less than that calculated by the Dirac equation. The calculation of the Lamb shift now forms an essential part in the theoretical calculations of energy levels and ionization energies of one and two electron atomic ions $[8,9]$.

In this work, we begin by showing the expressions with relativistic corrections to calculate ionization energies which give excellent agreement with the literature values. However, as the number of electrons in the sequence increases, the expressions become more and more complex and less predictable. Hence, we discuss alternative simple equations to calculate energies of isoelectronic sequences in excess of five electrons and show that it is more practical to adopt a simple expression to reproduce ionization energies which still provide very good agreement with generally accepted values. To maintain our aim of simplicity, the expressions/equations only contain fundamental constants or values derived from fundamental constants and fairly simple numbers.

\section{Sources of Data}

Moore [10-13] provided very detailed tables of atomic energy levels and ionization potentials in wave numbers $\left(\mathrm{cm}^{-1}\right)$ and values converted from wave numbers to electron volts 
$(\mathrm{eV})$ (where $1 \mathrm{~cm}^{-1}$ equals $1.2398418 \times 10^{-4} \mathrm{eV}$ ) for atoms and atomic ions with estimated experimental errors and references to original work. These remain the most extensive survey of ionization energies and are still quoted in recent publications. The CRC Handbook of Chemistry and Physics [14] contains comprehensive data from Moore and later sources but does not supply information on estimated uncertainties. The majority of published ionization energy data are now available on the National Institute of Standards and Technology web site (http://www.nist.gov/srd/). These compilations include values of ionization energies that are accurately measured as well as crude estimates.

\section{Ionization Energies of the Hydrogen to Boron Isoelectronic Sequences}

When an electron in a multielectron system is ionized, we assume that the ionization energy contains $\left(J_{k}\right)$ the kinetic energy term; $\left(J_{l}\right)$ the Lamb shift term, $\left(J_{t}\right)$ the relaxation/ transition energy term, and any residual interaction $\left(E_{r}\right)[2]$. Therefore, the ionization energy can be represented as [15]

$$
\frac{1}{n^{2}} \mu\left(J_{k}-J_{l}-J_{t}\right)+E_{r}=E_{k}-E_{l}-E_{t}+E_{r}
$$

where $n$ is the principal quantum number and $\mu$ is the reduced mass and are provided in Table 2. $E_{k}, E_{l}$, and $E_{t}$ represent $J_{k}$, $J_{l}$, and $J_{t}$ multiplied by $1 / n^{2} \mu$, respectively. $J_{k}, J_{l}$, and $J_{t}$ are multiplied by $1 / n^{2} \mu$ because the electron ionized occurs in energy level $n$ and it revolves around the centre of mass. A list of reduced masses are given in Table 2. It is common to present ionization energies in $\mathrm{eV}$ (electron volt). Calculated results in this work are converted to $\mathrm{eV}$ from Joules by using the relationship of $1 \mathrm{eV}=1.60217648 \times 10^{-19} \mathrm{~J}$, any other figures in $\mathrm{cm}^{-1}$ are converted to $\mathrm{eV}$ by multiplying them with the value 0.00012398418 .

\section{The Relativistic, Lamb Shift, Electron Transition/Relaxation, and Residual Corrections}

The energy of an electron moving in a Bohr orbit can be represented by

$$
\frac{m_{o} v_{o}^{2}}{a_{0}}=\frac{q_{1} q_{2}}{\left(4 \pi \varepsilon_{o} a_{0}^{2}\right)}
$$

where $m_{o}$ is the electron rest mass, $v_{o}$ is the velocity of the electron, $q_{1} q_{2}$ stand for the charges of the electron and nucleus, $\varepsilon_{o}$ is the permittivity of a vacuum, and $a_{0}$ is the Bohr radius. The velocity $v_{o}$ of the electron in the hydrogen atom can be calculated from the above relationship and is equal to $2.1876913 \times 10^{6} \mathrm{~m} / \mathrm{sec}$. The velocity $v$ of the electron in successive atoms of the one-electron series increases by $Z$ times where $Z$ is the atomic/proton number or $v=v_{o} Z$. When there is more than one electron in the system, we assume that the velocity of the electron changes by $(Z-S)$ where $S$ is the screening constant for that electron.
The theory of relativity [16] points out that the mass $m$ of a moving particle is given by the expression $m=$ $m_{o} /\left(\sqrt{\left(1-v^{2} / c^{2}\right)}\right)$ where $m_{o}$ is the rest mass of particle. Expansion of this expression gives

$$
m=m_{o}\left(1+\frac{1}{2} \frac{v^{2}}{c^{2}}+\frac{3}{8} \frac{v^{4}}{c^{4}}+\frac{5}{16} \frac{v^{6}}{c^{6}}+\cdots\right),
$$

therefore it follows that $(1 / 2) m v^{2}=(1 / 2) m_{o} v^{2}\left(1+(1 / 2) v^{2} /\right.$ $\left.c^{2}+(3 / 8) v^{4} / c^{4}+(5 / 16) v^{6} / c^{6}+\cdots\right)$.

The kinetic energy components including relativistic correction for a one-electron atom at an equilibrium position (when the relativistic correction is half that of the maximum) are then

$$
\frac{1}{2} m_{o} v^{2}+0.5\left(\frac{1}{4} m_{o} \frac{v^{4}}{c^{2}}+\frac{3}{16} m_{o} \frac{v^{6}}{c^{4}}\right)
$$

The ionization energy of a one-electron atom is then

$$
I=\mu\left(\frac{1}{2} m_{o} v^{2}+0.5\left(\frac{1}{4} m_{o} \frac{v^{4}}{c^{2}}+\frac{3}{16} m_{o} \frac{v^{6}}{c^{4}}\right)-E_{l}\right) .
$$

The Lamb shift is usually computed by highly complex formulas which require lengthy computer routines to compute [17]. We assume (without theoretical justification) that the Lamb shift is a relativistic charge, mass, and size ratio effect. The reduced mass calculation [18] implicitly assumes that the electron and nucleus are point charges. But they have a finite size hence there needs to be an extra component in the reduced mass calculation. The energy reduction to take account of reduced mass is not simply $m_{e} /\left(m_{e}+m_{p}\right)\left((1 / 2) m_{o} v_{o}{ }^{2}\right)$ but should include a function of the charge and the ratio of nuclear to atomic size, and this component is calculated by the following expression:

$$
E_{l}=\left(\frac{m_{e}}{m_{e}+m_{p}}\right)\left(\frac{\alpha}{2^{.67}}\right)\left(\frac{1}{2} m_{o} v_{o}^{2}\right)(Z-S)^{3.2} A^{1 / 3},
$$

where $m_{e}$ is the electron mass, $m_{p}$ is the proton mass, and the factor for the reduced mass correction for hydrogen is $m_{e} /\left(m_{e}+m_{p}\right)$. The size of the nucleus increases roughly proportional to $A^{1 / 3}[19] . \alpha$ is the fine structure constant, and $\left(\alpha / 2^{67}\right)$ is a crude approximation of the square root of the ratio of nuclear to atomic size for hydrogen, and $A$ is the mass number of the atom. In a one electron system, $S$ is zero.

After an electron is ionized, one or more of the remaining electron(s) is/are attracted more closely to the nucleus. The attractive energy between the proton and the remaining electron(s) changes because of the change in screening experienced by the remaining electron(s) before and after ionization, [15] and this transition/relaxation energy is a function of

$$
\frac{n^{2}}{4}\left(\frac{1}{2} m_{o}\left(v_{o}\left(Z-S_{1}\right)\right)^{2}-\frac{1}{2} m_{o}\left(v_{o}\left(Z-S_{2}\right)\right)^{2}\right),
$$

where $S_{1}$ is the screening constant for the remaining elec$\operatorname{tron}(\mathrm{s})$ after ionization and $S_{2}$ is the screening constant for the remaining electron(s) before ionization. 
In the helium system, there are two electrons and both occupy the 1s orbital. Since each electron occupies half of the space and each is repelled by only one other electron (here we have assumed that the two electrons act as in a two-particle problem and are equivalent), the screening constant is a half (0.5). After ionization, there is zero screening.

In the lithium series, the electron that is ionized occupies a higher (2s) orbital and is shielded by two 1s electrons, and the screening increases by 1 to 1.5 . The extra screening experienced by the two inner electrons in the 1s orbital increases to 0.625 and which is an increase by $1 / 8$ or 0.125 rather than 0.5 because the third electron occupies a different orbital and in a different electron shell (i.e., 0.5 of 0.5 of 0.5 ). After ionization, only two electrons are left in the system and the screening reduces to just 0.5 .

In the beryllium series, the electron that is ionized occupies the 2 s orbital. Since there are four electrons and each moving in an elliptical orbit, each may at any time interact differently with the other three electrons. The screening of the fourth electron increases by a half to 2 , and the other electron in the $2 \mathrm{~s}$ orbital experiences a screening of between 1.5 and 2 and is 1.75. After ionization of the fourth electron, the screening experienced by the third electron drops back to 1.5 .

For the boron system, the outermost electron occupies a new orbital and the screening increases by 1 to 3 . The screening of the fourth electron increases by 0.25 to 2.25 , and after ionization the screening reduces back to 2 .

We assume that there are two opposite and competing residual electron-electron interactions, and this is discussed in detail in previous work and is not fully reproduced here [15]. In summary, there are temporary asymmetric distributions of electrons (e.g., they may be nearer to each other or further apart than average). The first type (when they are nearer to each other) is residual electron-electron repulsion which reduces slightly the energy required to ionize the electron, and this reduction diminishes very rapidly because with each successive ionization the size of the electron orbit/shell becomes smaller and the electrons become much more tightly bound to the nucleus. The reduction in energy resulting from this interaction is expressed by

$$
E_{r 1}=\left(\frac{1}{2} m_{o} v_{o}^{2}\right) \frac{Q_{I} \alpha}{\sqrt{((Z-(I-1)) !)}}
$$

The opposite and competing electron-electron interaction occurs when there are more than two electrons in the system. In a three electron system, two electrons occupy the 1s orbital and one occupies the $2 \mathrm{~s}$ orbital. The instantaneous asymmetric distribution of electrons produces an effect which result in the $2 \mathrm{~s}$ electron being screened slightly less than 0.5 from each of the $1 \mathrm{~s}$ electrons. The result is that one of the electrons is temporarily attracted to the nucleus a bit more than expected and hence increases the amount of energy required to remove it from the atom/ion. This is represented by

$$
E_{r 2}=\frac{1}{n^{2}}\left(\frac{1}{2} m_{o} v_{o}^{2}(Z-S)^{2}\right) \frac{\alpha Q_{I I}}{2}
$$

TABLE 1: Symbols, conversion factors, and constants (to 9 significant figures).

\begin{tabular}{lc}
\hline Symbol/constant & Value/comment/definition \\
\hline$\alpha$ & 0.00729735254 \\
$\mathrm{eV}$ & Number of electrons remaining after ionization \\
$n$ & 1 electron volt $=1.60217648 \times 10^{-19}$ Joules \\
$v$ & Principal quantum number \\
$Q$ & Electron velocity \\
$A$ & Number of residual electron-electron \\
$\mu$ & interactions \\
$S$ & Mass number \\
$S_{1}$ & Reduced mass (see Table 8 for individual \\
& values) \\
$S_{2}$ & Screening constant for the ionizing electron \\
$Z$ & Screening for the remaining electron $(\mathrm{s}) \mathrm{before}$ \\
$c$ & ionization \\
$m_{e}$ & Proton number (nuclear charge) \\
$m_{p}$ & Velocity of light $2.99792458 \times 10^{8} \mathrm{~ms} \mathrm{~m}^{-1}$ \\
$q$ & Electron rest mass $9.10938215 \times 10^{-31} \mathrm{~kg}$ \\
$\varepsilon_{o}$ & Proton rest mass $1.67262164 \times 10^{-27} \mathrm{~kg}$ \\
\hline & Electron charge $1.60217649 \times 10^{-19} \mathrm{C}$ \\
& Planck's constant $6.62606896 \times 10^{-34} \mathrm{Js}$ \\
& $8.85418782 \times 10^{-12} \mathrm{Fm}^{-1}$ \\
\end{tabular}

and the total residual electron interaction energy change is

$$
\begin{aligned}
E_{r}=( & -\left(\frac{1}{2} m_{o} v_{o}^{2}\right) \frac{Q_{I} \alpha}{\sqrt{((Z-(I-1)) !)}} \\
& \left.+\frac{1}{n^{2}}\left(\frac{1}{2} m_{o} v_{o}^{2}(Z-S)^{2}\right) \frac{\alpha Q_{I I}}{2}\right) .
\end{aligned}
$$

Symbols and values of constants shown in all the expressions in this work are given in Table $1 . Q_{I}$ is the number of electron-electron interactions before ionization and $Q_{I I}$ is the number of electron-electron interactions after ionization.

\section{Ionization Energies of One and Two Electron Ions}

There is only one electron and $n$ is 1 , the formula for calculating the ionization energy is

$$
\begin{aligned}
I_{1}=\mu & \frac{1}{2} m_{o} v^{2}+0.5\left(\frac{1}{4} m_{o} \frac{v^{4}}{c^{2}}+\frac{3}{16} m_{o} \frac{v^{6}}{c^{4}}\right) \\
& \left.-\left(\frac{m_{e}}{m_{e}+m_{p}}\right)\left(\frac{\alpha}{2^{67}}\right)\left(\frac{1}{2} m_{o} v_{o}^{2}\right)(Z)^{3.2} A^{1 / 3}\right) .
\end{aligned}
$$


TABLE 2: Coefficients/constants for calculating ionization energies of multielectron ions.

\begin{tabular}{|c|c|c|c|c|}
\hline \multirow{2}{*}{$Z$} & (1) & (2) & (3) & $(4)$ \\
\hline & Screening constants & Coefficient $a$ & Coefficient $b$ & Reduced mass* \\
\hline B & 3 & 0.318 & 0.032 & 0.999939550 \\
\hline $\mathrm{C}$ & 3.5 & 0.572 & 0.490 & 0.999954670 \\
\hline $\mathrm{N}$ & 4 & 0.842 & 1.170 & 0.999961152 \\
\hline $\mathrm{O}$ & 5 & 0.716 & 0.731 & 0.999966015 \\
\hline $\mathrm{F}$ & 5.5 & 1.054 & 2.357 & 0.999971388 \\
\hline $\mathrm{Ne}$ & 6 & 1.318 & 3.520 & 0.999972825 \\
\hline $\mathrm{Na}$ & 7 & 2.222 & 11.809 & 0.999976377 \\
\hline $\mathrm{Mg}$ & 7.5 & 2.344 & 12.925 & 0.999977367 \\
\hline $\mathrm{Al}$ & 8.5 & 2.882 & 21.124 & 0.999979889 \\
\hline $\mathrm{Si}$ & 9 & 3.012 & 22.521 & 0.999980614 \\
\hline $\mathrm{P}$ & 9.5 & 3.194 & 24.599 & 0.999982497 \\
\hline S & 10.5 & 2.850 & 22.194 & 0.999983051 \\
\hline $\mathrm{Cl}$ & 11 & 2.968 & 23.026 & 0.999983571 \\
\hline $\mathrm{Ar}$ & 11.5 & 3.306 & 27.683 & 0.999986454 \\
\hline ** & Screening constants & Coefficient $a$ & Coefficient $b$ & Reduced mass \\
\hline $\mathrm{K}$ & 11 & 2.968 & 23.026 & 0.999986113 \\
\hline $\mathrm{Ca}$ & 11.5 & 3.306 & 27.683 & 0.999986467 \\
\hline $\mathrm{Sc}$ & 13.5 & 3.366 & 30.828 & 0.999988489 \\
\hline $\mathrm{Ti}$ & 14 & 3.832 & 38.481 & 0.999989186 \\
\hline $\mathrm{V}$ & 14.5 & 4.542 & 51.618 & 0.999989405 \\
\hline $\mathrm{Cr}$ & 15 & 5.138 & 62.763 & 0.999990000 \\
\hline $\mathrm{Mn}$ & 15.5 & 5.658 & 73.472 & 0.999990188 \\
\hline $\mathrm{Fe}$ & 16.5 & 5.850 & 82.119 & 0.999990702 \\
\hline Co & 17 & 4.484 & 57.749 & 0.999990708 \\
\hline
\end{tabular}

${ }^{*}$ Reduced masses are calculated from atomic number 5 to atomic number 26; for ions above atomic number 26 , the reduced mass of atomic 26 is used since the differences between reduced masses at high atomic numbers are too small to show any differences in the calculated results.

** From the potassium series, the constants apply to calculating ionization energies from the third ionization energy (i.e., third ionization of potassium, fourth ionization of calcium, etc.).

The one-electron ionization energies calculated by (11) when compared with the ionization energies published in the CRC Handbook of Chemistry and Physics agree to $99.999 \%$ or better in the majority of cases. The biggest absolute difference is $0.086 \mathrm{eV}$ (to 3 decimal places) from a calculated value of $5469.95 \mathrm{eV}$ or $0.00164 \%$ [15].

The ionization energy of a two electron system is $\left(E_{k}-\right.$ $\left.E_{l}-E_{t}+E_{r}\right)$, where

$$
\begin{gathered}
E_{l}=\mu\left(\left(\frac{m_{e}}{m_{e}+m_{p}}\right)\left(\frac{\alpha}{2^{2 / 3}}\right)\left(\frac{1}{2} m_{o} v_{o}^{2}\right)(Z-0.5)^{3.2} A^{1 / 3}\right) ; \\
E_{t}=0.25 \mu\left(\frac{1}{2} m_{o}\left(v_{o}(Z)\right)^{2}-\frac{1}{2} m_{o}\left(v_{o}(Z-0.5)\right)^{2}\right) ; \\
E_{r}=\left(-\left(\frac{1}{2} m_{o} v_{o}^{2}\right) \frac{\alpha}{\sqrt{((Z) !)}}\right) .
\end{gathered}
$$

Since, for simplicity, we have not applied a relativistic correction to $E_{t}$ we have made a crude approximation of reducing the relativistic correction in $E_{k}$ by another $5 \%$ to 0.45 (rather than 0.5$)$ and where $v$ is $\left(v_{o}(Z-0.5)\right)$, so it is

$$
E_{k}=\mu\left(\frac{1}{2} m_{o} v^{2}+0.45\left(\frac{1}{4} m_{o} \frac{v^{4}}{c^{2}}+\frac{3}{16} m_{o} \frac{v^{6}}{c^{4}}\right)\right) .
$$

When compared to the CRC Handbook of Chemistry and Physics the majority of values differ by less than $0.01 \%$, the largest absolute difference is $0.216 \mathrm{eV}$ from a calculated value of $2437.846 \mathrm{eV}$ or $0.0089 \%$.

\section{Ionization Energies of Three-, Four-, and Five-Electron Ions}

The ionization energy of a three-, four-, and five electron system is

$$
\left(E_{k}-E_{l}-E_{t}+E_{r}\right)
$$


where

$$
\begin{gathered}
E_{l}=0.25 \mu\left(\left(\frac{m_{e}}{m_{e}+m_{p}}\right)\left(\frac{\alpha}{2^{2 / 3}}\right)\left(\frac{1}{2} m_{o} v_{o}^{2}\right)(Z-0.5)^{3.2} A^{1 / 3}\right) ; \\
E_{t}=\mu\left(\frac{1}{2} m_{o}\left(v_{o}\left(Z-S_{1}\right)\right)^{2}-\frac{1}{2} m_{o}\left(v_{o}\left(Z-S_{2}\right)\right)^{2}\right) ; \\
E_{r}=\left(\left(-\left(\frac{1}{2} m_{o} v_{o}^{2}\right) \frac{Q_{I} \alpha}{\sqrt{((Z-(I-1)) !)}}\right.\right. \\
\left.\left.+\frac{1}{n^{2}}\left(\frac{1}{2} m_{o} v_{o}^{2}(Z-S)^{2}\right) \frac{\alpha Q_{I I}}{2}\right)\right) .
\end{gathered}
$$

As with the two-electron system, we have not applied a relativistic correction to $E_{t}$ but we have made a crude approximation of reducing the relativistic correction in $E_{k}$ by $5 \%$ to 0.45 and where $v$ is $\left(v_{o}(Z-S)\right)$, so that the expression becomes

$$
E_{k}=0.25 \mu\left(\frac{1}{2} m_{o} v^{2}+0.45\left(\frac{1}{4} m_{o} \frac{v^{4}}{c^{2}}+\frac{3}{16} m_{o} \frac{v^{6}}{c^{4}}\right)\right) .
$$

The agreement with the literature values of ionization energies is $99 \%$ or better in all cases. It is evident from the above discussion that as the number of electrons in the system increases the equations get more complicated and it is difficult to determine the various corrections.

\section{Alternative Simple Equations to Calculate Ionization Energies}

Besides the more complicated equations shown above, ionization energies can be calculated with simpler expressions but not so precise. A simple formula is sometimes used to calculate ionization energies. It often takes the form of [20]

$$
I=\left(\frac{1}{n^{* 2}}\right) h c R_{H}(Z-S)^{2}
$$

where $R_{H}$ is the Rydberg constant for hydrogen $\left(R_{\infty}\right.$, the Rydberg constant for infinite mass is equivalent to $13.6059 \mathrm{eV}), n^{*}$ is an "effective" quantum number, $Z$ is the atomic number, and $S$ is the screening constant based on Slater's rules [21] which enable approximations of analytic wave functions to be constructed for rough estimates [22]. Equation (17) makes a very simplistic assumption that when an electron is removed from an atom or ion, the atom/ion remains unchanged except for the removal of that electron. We believe that it is incorrect to use equations like (17) (where the ionization energy is considered as a function of a complete square) to calculate energies in isoelectronic series. For multielectron systems, we need to consider electron transition/relaxation and other smaller components which may influence the ionization energy of an electron.

In a multielectron system, the main components of the energy change during ionization are the electron-proton energy or $(Z-S)^{2}$ and the electron relaxation energy (or $k n^{2}\left[\left(\left(Z-S_{1}\right)^{2}-\left(Z-S_{2}\right)^{2}\right)\right]$ where $k$ is a constant dependent on the particular shell/orbital, $S_{1}$ and $S_{2}$ represent the screening constants of the remaining electrons after and before the electron is ionized). In most cases, the electron-proton energy component alone accounts for $90 \%$ or more of the energy change and together with the relaxation energy can represent more than $95 \%$ of the total energy change. If factors which in total contribute only a small percentage of the energy change, such as residual repulsion, pairing or exchange energies are excluded, the expression for calculating the ionization energy can be approximated to

$$
\begin{aligned}
I= & \left(\frac{1}{n^{2}}\right) R_{\mu} h c \\
& \times\left\{(Z-S)^{2}-0.25 n^{2}\left[\left(\left(Z-S_{1}\right)^{2}-\left(Z-S_{2}\right)^{2}\right)\right]\right\} .
\end{aligned}
$$

This can be expanded to become

$$
\begin{aligned}
& I=\left(\frac{1}{n^{2}}\right) R_{\mu} h c\{(\left.Z^{2}-2 Z S+S^{2}\right) \\
&-k\left[\left(Z^{2}-2 Z S_{1}+S_{1}^{2}\right)\right. \\
&\left.\left.-\left(Z^{2}-2 Z S_{2}+S_{2}^{2}\right)\right]\right\} .
\end{aligned}
$$

Since in the second half of expression (19) the $Z^{2}$ term cancels out, only $2 Z\left(S_{1}-S_{2}\right)$ and $\left(S_{2}^{2}-S_{1}^{2}\right)$ are left. $2 Z\left(S_{1}-S_{2}\right)$ can be reduced to $a Z$, and $\left(S_{2}{ }^{2}-S_{1}{ }^{2}\right)$ becomes a constant $b$. This can be rearranged and simplified to

$$
I=\left(\frac{1}{n^{2}}\right) R_{\mu} h c\left\{\left(Z^{2}-2 Z S+S^{2}\right)-a Z+b\right\},
$$

where $a$ and $b$ are constants for each isoelectronic system, $n$ is the principal quantum number, and, unlike (17), expression (20) is not a complete square.

We have formulated the following rules for working out screening (shielding) constants: (1) for each additional electron in the system, the screening increases by 0.5 unless (2) the electron to be ionized occupies a new orbital such as from beryllium to boron when it increases by 1 and (3) to account for the pairing effect, such as from nitrogen to oxygen, the screening constant increases by 1 . For example, for the carbon system, the screening increases by 0.5 units to 3.5 and increases by a further 0.5 to 4 for nitrogen but increases to 5 for oxygen.

We made different estimates of the screening constants $S_{2}$ and $S_{1}$ and obtained various values of $a$ and $b$. We then selected the values that give the best results and a list of screening constants, coefficients $a$ and $b$ and reduced masses as shown in Table 2. They are used with equation (20) to calculated the ionization energies of isoelectronic sequences from five electrons.

The values calculated from expression (20) are presented for the first six appropriate members of each series for the following two reasons. Firstly, as we have already shown [14], ionization energies of the first few members of isoelectronic sequences are the most precise. Sometimes, only the first four or five members of a series are experimentally measured and uncertainties increase further along a series. 
TABle 3: Ionization energies (eV) of isoelectronic series from the CRC Handbook (5 to 18 electron sequences) - first six members of each series.

\begin{tabular}{|c|c|c|c|c|c|c|}
\hline \multirow{2}{*}{$Z$} & $(2)$ & (3) & $(4)$ & (5) & (6) & (7) \\
\hline & First & Second & Third & Fourth & Fifth & Sixth \\
\hline B & 8.298 & 24.383 & 47.449 & 77.414 & 114.243 & 157.930 \\
\hline $\mathrm{C}$ & 11.260 & 29.601 & 54.936 & 87.140 & 126.210 & 172.180 \\
\hline $\mathrm{N}$ & 14.534 & 35.121 & 62.708 & 97.120 & 138.400 & 186.760 \\
\hline $\mathrm{O}$ & 13.618 & 34.971 & 63.450 & 98.910 & 141.270 & 190.490 \\
\hline $\mathrm{F}$ & 17.423 & 40.963 & 71.620 & 109.266 & 153.825 & 205.270 \\
\hline $\mathrm{Ne}$ & 21.565 & 47.286 & 80.144 & 119.992 & 166.767 & 220.421 \\
\hline $\mathrm{Na}$ & 5.139 & 15.035 & 28.448 & 45.142 & 65.025 & 88.053 \\
\hline $\mathrm{Mg}$ & 7.646 & 18.829 & 33.493 & 51.444 & 72.595 & 97.030 \\
\hline $\mathrm{Al}$ & 5.896 & 16.346 & 30.203 & 47.222 & 67.800 & 91.009 \\
\hline $\mathrm{Si}$ & 8.152 & 19.770 & 34.790 & 53.465 & 75.020 & 99.400 \\
\hline $\mathrm{P}$ & 10.487 & 23.338 & 39.610 & 59.810 & 82.660 & 108.780 \\
\hline S & 10.360 & 23.814 & 40.740 & 60.910 & 84.500 & 110.680 \\
\hline $\mathrm{Cl}$ & 12.968 & 27.630 & 45.806 & 67.270 & 91.650 & 119.530 \\
\hline $\mathrm{Ar}$ & 15.760 & 31.630 & 50.913 & 73.489 & 99.300 & 128.130 \\
\hline
\end{tabular}

TABLE 4: Ionization energies (eV) of isoelectronic series calculated using expression 23 and coefficients/constants from Table 2 (5 to 18 electron sequences).

\begin{tabular}{|c|c|c|c|c|c|c|}
\hline$Z$ & $(2)$ & (3) & $(4)$ & (5) & $(6)$ & $(7)$ \\
\hline & First & Second & Third & Fourth & Fifth & Sixth \\
\hline B & 8.306 & 24.231 & 46.959 & 76.490 & 112.824 & 155.960 \\
\hline $\mathrm{C}$ & 11.252 & 29.715 & 54.980 & 87.049 & 125.920 & 171.595 \\
\hline $\mathrm{N}$ & 14.544 & 35.490 & 63.238 & 97.790 & 139.144 & 187.302 \\
\hline $\mathrm{O}$ & 13.616 & 34.990 & 63.167 & 98.148 & 139.930 & 188.517 \\
\hline $\mathrm{F}$ & 17.419 & 41.045 & 71.475 & 108.706 & 152.741 & 203.579 \\
\hline $\mathrm{Ne}$ & 21.564 & 47.693 & 80.626 & 120.361 & 166.900 & 220.241 \\
\hline $\mathrm{Na}$ & 5.090 & 15.336 & 28.606 & 44.900 & 64.217 & 86.558 \\
\hline $\mathrm{Mg}$ & 7.629 & 19.203 & 33.801 & 51.422 & 72.066 & 95.734 \\
\hline $\mathrm{Al}$ & 5.907 & 16.668 & 30.452 & 47.260 & 67.091 & 89.945 \\
\hline $\mathrm{Si}$ & 8.092 & 20.168 & 35.267 & 53.390 & 74.536 & 98.706 \\
\hline $\mathrm{P}$ & 10.489 & 23.802 & 40.138 & 59.497 & 81.880 & 107.287 \\
\hline$S$ & 10.347 & 24.179 & 41.035 & 60.915 & 83.818 & 109.745 \\
\hline $\mathrm{Cl}$ & 12.955 & 28.121 & 46.310 & 67.523 & 91.760 & 119.020 \\
\hline $\mathrm{Ar}$ & 15.760 & 31.926 & 51.116 & 73.330 & 98.567 & 126.828 \\
\hline
\end{tabular}

Secondly, our results are compared with ionization energies with those compiled in the CRC Handbook of Chemistry and Physics, and for many isoelectronic series with more than twenty electrons, only a limited number of values are available for each sequence. Some of these values are given to many significant figures and some only to two or three significant figures because uncertainties can be of the order of $1 \mathrm{eV}$ or higher. Since, as with previous work, all our results are rounded to three decimal places we have decided that where the CRC Handbook of Chemistry and Physics has provided values with more than three decimal places they are rounded to three decimal places in the tables.

\section{Ionization Energies from Five- to Eighteen-Electron Isoelectronic Atomic Ions}

Ionization energies reported in the CRC Handbook for five to eighteen electronic series (first six members) are given in Table 3. Values of ionization energies calculated using our coefficients are provided in Table 4. Percentage differences between our values and values published by 
Table 5: Percentage difference between values shown in Tables 3 and 4 .

\begin{tabular}{|c|c|c|c|c|c|c|}
\hline \multirow{2}{*}{$Z$} & (2) & (3) & (4) & (5) & (6) & (7) \\
\hline & First & Second & Third & Fourth & Fifth & Sixth \\
\hline B & -0.1 & 0.6 & 1.0 & 1.2 & 1.2 & 1.2 \\
\hline C & 0.1 & -0.4 & -0.1 & 0.1 & 0.2 & 0.3 \\
\hline $\mathrm{N}$ & -0.1 & -1.0 & -0.8 & -0.7 & -0.5 & -0.3 \\
\hline $\mathrm{O}$ & 0.0 & -0.1 & 0.4 & 0.8 & 0.9 & 1.0 \\
\hline $\mathrm{F}$ & 0.0 & -0.2 & 0.2 & 0.5 & 0.7 & 0.8 \\
\hline $\mathrm{Ne}$ & 0.0 & -0.9 & -0.6 & -0.3 & -0.1 & 0.1 \\
\hline $\mathrm{Na}$ & 1.0 & -2.0 & -0.6 & 0.5 & 1.2 & 1.7 \\
\hline $\mathrm{Mg}$ & 0.2 & -2.0 & -0.9 & 0.0 & 0.7 & 1.3 \\
\hline $\mathrm{Al}$ & 1.3 & -2.0 & -0.8 & -0.1 & 1.0 & 1.2 \\
\hline $\mathrm{Si}$ & 0.7 & -2.0 & -1.4 & 0.1 & 0.6 & 0.7 \\
\hline $\mathrm{P}$ & 0.0 & -2.0 & -1.3 & 0.5 & 0.9 & 1.4 \\
\hline S & 0.1 & -1.5 & -0.7 & 0.0 & 0.8 & 0.8 \\
\hline $\mathrm{Cl}$ & 0.1 & -1.8 & -1.1 & -0.4 & -0.1 & 0.4 \\
\hline $\mathrm{Ar}$ & 0.0 & -0.9 & -0.4 & 0.2 & 0.7 & 1.0 \\
\hline
\end{tabular}

the $C R C$ Handbook as listed in Table 5 show that all values agree to $98 \%$ or better. Just over $76 \%$, the calculated values agree to $99 \%$ or better.

\section{Ionization Energies from Nineteen- to Twenty-Seven-Electron Isoelectronic Sequences}

Treatment of ionization energies of isoelectronic with nineteen electrons or more are different and more complicated. This is because, from atomic number nineteen, the electron to be ionized is in period 4 of the periodic table and such an isoelectronic system includes ionization of $\mathrm{s}$ or d electrons [23]. We have demonstrated that for the transition metals or lanthanide [24] elements, the ionization process is complicated and, in the majority of cases, $\mathrm{d}$ or $\mathrm{f}$ electrons are not removed in the first or second ionization. For example, consider the potassium, calcium, scandium, and titanium series (isoelectronic series with 19, 20, 21, and 22 electrons resp.). For the potassium series (19-electron isoelectronic), the first ionization of potassium and the second ionization of calcium both involve removal of a 4 s electron. But the third ionization of scandium and the fourth ionization of titanium (which are in the same isoelectronic series) involve removal of a $3 \mathrm{~d}$ electron. For the calcium series (20-electron isoelectronic), the first ionization of calcium and the second ionization of scandium involve removal of a 4 s electron whereas the third ionization of titanium involves removal of a $3 \mathrm{~d}$ electron. Similarly with the scandium and titanium series, for the first two members of the series, the energy change is the energy required to ionize a $4 \mathrm{~s}$ electron but from the third member of the series onwards the energy change is the energy for ionizing a $3 \mathrm{~d}$ electron (a more detailed discussion is provided in a previous work [23]). Equation (20) shows that ionization is a function of $1 / n^{2}$. Therefore, for the $19,20,21$, and 22 isoelectronic series, the correct fraction or decimal for $1 / n^{2}$ to use is $1 / 16$ (or 0.0625 ) for the first two members of the series but $1 / 9$ (or 0.11111 ) for the remainder of the series. Hence, for the above reasons, it is incorrect to use a single set of coefficients to calculate the energies of an isoelectronic sequence which may involve removal of electrons in different orbitals.

We believe that there is little value calculating the first and second ionization energies of sequences beginning with the potassium. From the potassium series onwards, the third ionization energy requires a different set of coefficients. For example, for the potassium series, a set of coefficients is used to calculate the third ionization energy of potassium, the fourth ionization energy of calcium, and the fifth ionization energy of scandium, and so on because a $3 p$ electron is ionized in all cases. For the scandium series, the third ionization of scandium, the fourth ionization of titanium, and the fifth ionization of vanadium and so on can be calculated using one set of coefficients since in all cases a $3 \mathrm{~d}$ electron is ionized. However, the third ionization energy of the potassium series and the third ionization energy of the calcium series are identical to the fifth ionization of the chlorine and argon series, respectively. Hence, these two series will not be repeated in Tables 6 and 7 .

We have calculated the ionization energies up to the cobalt series (which contains five appropriate published values for comparison) because beyond the cobalt series there are fewer and fewer published values available for use in comparison. Ionization energies of isoelectronic series reported in the CRC Handbook for sequences from scandium to cobalt (for each series beginning with the third ionization energy) are given in Table 6 . Values of ionization energies calculated using our coefficients are provided in Table 7. Percentage differences between our values and values in the CRC Handbook as listed in Table 8 show that all calculated values agree to $98 \%$, or better and just under $83 \%$ of the values agree to $99 \%$ or better. 
TABLE 6: Ionization energies (eV) of isoelectronic series from the CRC.

\begin{tabular}{|c|c|c|c|c|c|c|}
\hline \multirow{2}{*}{$Z$} & (2) & (3) & (4) & (5) & (6) & (7) \\
\hline & Third & Fourth & Fifth & Sixth & Seventh & Eighth \\
\hline Sc & 24.757 & 43.267 & 65.282 & 90.635 & 119.203 & 151.060 \\
\hline $\mathrm{Ti}$ & 27.492 & 46.709 & 69.460 & 95.600 & 124.980 & 157.800 \\
\hline $\mathrm{V}$ & 29.311 & 49.160 & 72.400 & 99.100 & 128.900 & 162.000 \\
\hline $\mathrm{Cr}$ & 30.960 & 51.200 & 75.000 & 102.000 & 133.000 & 166.000 \\
\hline $\mathrm{Mn}$ & 33.668 & 54.800 & 79.500 & 108.000 & 139.000 & 174.000 \\
\hline $\mathrm{Fe}$ & 30.652 & 51.300 & 76.060 & 103.000 & 134.000 & 169.900 \\
\hline Co & 33.500 & 54.900 & 79.800 & 108.000 & 140.900 & N/A \\
\hline
\end{tabular}

TABLE 7: Ionization energies (eV) of isoelectronic series using (20) and constants/coefficients in Table $2^{*}$.

\begin{tabular}{|c|c|c|c|c|c|c|}
\hline \multirow{2}{*}{$Z$} & $(2)$ & (3) & $(4)$ & (5) & (6) & $(7)$ \\
\hline & Third & Fourth & Fifth & Sixth & Seventh & Eighth \\
\hline Sc & 24.780 & 43.879 & 66.002 & 91.149 & 119.319 & 150.513 \\
\hline $\mathrm{Ti}$ & 27.479 & 47.386 & 70.316 & 96.270 & 125.247 & 157.248 \\
\hline $\mathrm{V}$ & 29.330 & 49.676 & 73.044 & 99.436 & 128.852 & 161.291 \\
\hline $\mathrm{Cr}$ & 30.917 & 51.873 & 75.853 & 102.856 & 132.882 & 165.932 \\
\hline $\mathrm{Mn}$ & 33.670 & 55.351 & 80.056 & 107.785 & 138.537 & 172.313 \\
\hline $\mathrm{Fe}$ & 30.642 & 52.034 & 76.448 & 103.887 & 134.349 & 167.834 \\
\hline Co & 33.509 & 55.453 & 80.421 & 108.413 & 139.428 & N/A \\
\hline
\end{tabular}

${ }^{*}$ Ionization energy of isoelectronic series starting from the third ionization energy of the appropriate series.

TABLE 8: Percentage difference between values shown in Tables 6 and 7.

\begin{tabular}{lcccccc}
\hline$Z$ & $(2)$ & $(3)$ & $(4)$ & $(5)$ & $(6)$ & $(7)$ \\
& Third & Fourth & Fifth & Sixth & Seventh & Eighth \\
\hline Sc & -0.1 & -1.4 & -1.1 & -0.6 & -0.1 & 0.4 \\
$\mathrm{Ti}$ & 0.0 & -1.4 & -1.2 & -0.7 & -0.2 & 0.3 \\
$\mathrm{~V}$ & -0.1 & -1.0 & -0.9 & -0.3 & 0.0 & 0.4 \\
$\mathrm{Cr}$ & 0.1 & -1.3 & -1.1 & -0.8 & 0.1 & 0.0 \\
$\mathrm{Mn}$ & 0.0 & -1.0 & -0.7 & 0.2 & 0.3 & 1.0 \\
$\mathrm{Fe}$ & 0.0 & -1.4 & -0.5 & -0.9 & -0.3 & 1.2 \\
$\mathrm{Co}$ & 0.0 & -1.0 & -0.8 & -0.4 & 1.0 & N/A \\
\hline
\end{tabular}

\section{Discussion}

We have used a simple quadratic expression in this work. We have not considered exchange and orbital energies (20) and have ignored any residual interactions or relativistic corrections, which for multielectron systems are difficult to apply. Hence, it is not surprising that the agreement with some of the generally accepted values is less than $99 \%$. However, some of the differences between the calculated values and CRC Handbook values are less than the experimental uncertainties. However, as we have shown above, equations for solving ionization energies can be very complicated and the results may be unpredictable as the number of electrons in an isoelectronic series increases. Therefore, we believe that there is a strong case to use a simple quadratic expression rather than trying to create complex equations to calculate ionization energies.
TABle 9: Coefficients/constants proposed by Agmon to calculate ionization energies of isoelectronic sequences.

\begin{tabular}{lcc}
\hline$Z$ & $(2)$ & $(3)$ \\
& $n^{*}$ & $S$ \\
\hline $\mathrm{B}$ & 1.961 & 3.36 \\
$\mathrm{C}$ & 1.954 & 4.09 \\
$\mathrm{~N}$ & 1.952 & 4.82 \\
$\mathrm{O}$ & 1.934 & 5.83 \\
$\mathrm{~F}$ & 1.930 & 6.57 \\
$\mathrm{Ne}$ & 1.930 & 7.32 \\
$\mathrm{Na}$ & 2.865 & 8.78 \\
$\mathrm{Mg}$ & 2.851 & 9.46 \\
$\mathrm{Al}$ & 2.849 & 10.70 \\
$\mathrm{Si}$ & 2.835 & 11.40 \\
$\mathrm{P}$ & 2.814 & 12.13 \\
$\mathrm{~S}$ & 2.797 & 13.10 \\
$\mathrm{Cl}$ & 2.770 & 13.88 \\
$\mathrm{Ar}$ & 2.737 & 14.68 \\
\hline
\end{tabular}

Although Slater's rules are still cited in recent publications [25] as adequate for predicting most periodic trends, it has been pointed out that the rules are unreliable when orbitals with a total quantum number of 4 [26] is reached (e.g., a 3p orbital has a principal quantum number of 3 , orbital quantum number of 1 , and magnetic quantum number of 1 , and spin quantum number of $1 / 2$ already has a total quantum number of 5). Equation (17) and Slater's rules are based on simple assumptions and are unable to account for many different features of ionization energies across the periodic table. We 
TABLE 10: Ionization energies (eV) calculated using coefficients in Table 9.

\begin{tabular}{|c|c|c|c|c|c|c|}
\hline \multirow{2}{*}{$Z$} & (2) & (3) & (4) & (5) & (6) & (7) \\
\hline & First & Second & Third & Fourth & Fifth & Sixth \\
\hline B & 9.511 & 24.645 & 46.851 & 76.130 & 112.481 & 155.904 \\
\hline C & 12.992 & 30.159 & 54.448 & 85.860 & 124.394 & 170.052 \\
\hline $\mathrm{N}$ & 16.960 & 36.089 & 62.355 & 95.758 & 136.299 & 183.977 \\
\hline $\mathrm{O}$ & 17.119 & 36.533 & 63.217 & 97.172 & 138.399 & 186.896 \\
\hline $\mathrm{F}$ & 21.556 & 42.949 & 71.642 & 107.637 & 150.932 & 201.529 \\
\hline $\mathrm{Ne}$ & 26.220 & 49.437 & 79.956 & 117.776 & 162.897 & 215.319 \\
\hline $\mathrm{Na}$ & 8.165 & 17.177 & 29.502 & 45.141 & 64.092 & 86.358 \\
\hline $\mathrm{Mg}$ & 10.793 & 20.965 & 34.482 & 51.345 & 71.554 & 95.109 \\
\hline $\mathrm{Al}$ & 8.862 & 18.244 & 30.976 & 47.059 & 66.492 & 89.276 \\
\hline $\mathrm{Si}$ & 11.437 & 21.927 & 35.800 & 53.057 & 73.698 & 97.723 \\
\hline $\mathrm{P}$ & 14.145 & 25.719 & 40.727 & 59.170 & 81.048 & 106.360 \\
\hline S & 14.618 & 26.437 & 41.733 & 60.505 & 82.754 & 108.479 \\
\hline $\mathrm{Cl}$ & 17.251 & 30.082 & 46.457 & 66.377 & 89.841 & 116.850 \\
\hline $\mathrm{Ar}$ & 20.008 & 33.876 & 51.375 & 72.504 & 97.263 & 125.653 \\
\hline
\end{tabular}

TABLE 11: Percentage differences between results shown in Table 10 and values from the CRC Handbook.

\begin{tabular}{|c|c|c|c|c|c|c|}
\hline \multirow{2}{*}{ Z } & (2) & (3) & $(4)$ & (5) & (6) & $(7)$ \\
\hline & First & Second & Third & Fourth & Fifth & Sixth \\
\hline B & -14.6 & -1.1 & 1.3 & 1.7 & 1.5 & 1.3 \\
\hline $\mathrm{C}$ & -15.4 & -1.9 & 0.9 & 1.5 & 1.4 & 1.3 \\
\hline $\mathrm{N}$ & -16.7 & -2.8 & 0.6 & 1.4 & 1.5 & 1.5 \\
\hline $\mathrm{O}$ & -25.7 & -4.5 & 0.4 & 1.8 & 2.0 & 1.9 \\
\hline $\mathrm{F}$ & -23.7 & -4.8 & 0.0 & 1.5 & 1.9 & 1.8 \\
\hline $\mathrm{Ne}$ & -21.6 & -4.5 & 0.2 & 1.8 & 2.3 & 2.3 \\
\hline $\mathrm{Na}$ & -58.9 & -14.2 & -3.7 & 0.0 & 1.4 & 1.9 \\
\hline $\mathrm{Mg}$ & -41.2 & -11.3 & -2.9 & 0.8 & 1.8 & 1.7 \\
\hline $\mathrm{Al}$ & -48.1 & -11.6 & -2.6 & 0.3 & 1.9 & 1.9 \\
\hline $\mathrm{Si}$ & -40.3 & -10.9 & -2.9 & 0.8 & 1.8 & 1.7 \\
\hline $\mathrm{P}$ & -34.9 & -10.2 & -2.8 & 1.1 & 1.9 & 2.2 \\
\hline S & -41.1 & -11.0 & -2.4 & 0.7 & 2.1 & 2.0 \\
\hline $\mathrm{Cl}$ & -33.0 & -8.9 & -1.4 & 1.3 & 2.0 & 2.2 \\
\hline $\mathrm{Ar}$ & -26.9 & -7.1 & -0.9 & 1.3 & 2.1 & 1.9 \\
\hline
\end{tabular}

have also shown that ionization energies are not functions of simple complete squares [23], and Slater's rules cannot account for the complex patterns in ionization energies shown in our previous work [24].

\section{Conclusion}

Ionization energies calculated by a kinetic energy approach with simple relativistic and Lamb shift corrections give remarkable agreement with generally accepted values for one- to five-electron isoelectronic series. However, for multielectron isoelectronic series with five or more electrons, there is no accepted methodology for calculating relativistic corrections. Therefore, it is practical and more manageable to use a simple quadratic expression to calculate ionization energies which still give very good agreement with generally accepted values. We have not attempted to calculate ionization energies of sequences beyond cobalt because there are few sequences where a long series of data are available. We believe that expression (20) can be used to calculate ionization energies of any multielectron isoelectronic series assuming that sensible coefficients are applied. It is evident that an equation based on (17) (or function of a complete square such as $(Z-S)^{2}$ ) is not the correct representation of the energy change when an electron is ionized. We have also demonstrated that it is incorrect to use a single set of constants/coefficients to calculate the ionization energies of some isoelectronic series such as the potassium, calcium, or scandium series.

Since a great many of the experimental measurements of ionization energies were done over half a century ago, and some of the published values are extrapolated/interpolated, or estimates with fairly large uncertainties there is also a strong case for new measurements to be undertaken and reliability of some of the currently accepted values to be reexamined. 


\section{Appendix}

We believe it is useful to compare our results with other calculated results (using an equation which is a complete square) to show that our simple model of electron ionization is more realistic and reliable. Agmon [20] published a list of coefficients for the following equation to calculate ionization energies of isoelectronic series:

$$
E=\frac{h c R_{H}(Z-S)^{2}}{n^{* 2}},
$$

where $R_{H}$ is the Rydberg constant for hydrogen. $Z$ is the atomic number, $S$ is the screening constant, and $n^{*}$ is an "effective" quantum number.

The list is shown in Table 9. Ionization energies (for five- to eighteen-electron sequences) calculated by the above equation with the set of coefficients are shown in Table 10. Percentage differences with those in the CRC Handbook are shown in Table 11.

We have shown that, in general, ionization energies of the first few members (usually the first two or three) of an isoelectronic sequence are most accurately determined, uncertainties normally increase across the higher members of a series. It is clear from Table 10 that the biggest differences between the calculated and accepted values occur where the accuracy and reliability of the accepted values are the greatest. Difference between the calculated and generally accepted values for some of ionization energies are greater than $10 \%$. Secondly, only $13.1 \%$ of the calculated figures agree with the accepted ones to $99 \%$ or better (as compared to a much higher percentage of ours) and agreement of less (or worse) than 95\% occur with over $29.7 \%$ of the calculated figures (whereas all our results agree to $95 \%$ or better). A major factor is that values of ionization energies are not functions of complete squares, and the equation used by Agmon does not take account of the electron relaxation/transition energy.

\section{References}

[1] P. F. Lang and B. C. Smith, "Ionisation potentials of one-electron atoms," Inorganic and Nuclear Chemistry Letters, vol. 17, no. 1-2, pp. 27-29, 1981.

[2] M. E. S. Ali, P. F. Lang, and B. C. Smith, "Ionisation energies of two-electron atoms," Journal of the Chemical Society, Faraday Transactions 2, vol. 80, no. 9, pp. 1089-1091, 1984.

[3] P. F. Lang and B. C. Smith, "A simple formula to calculate the ionization energies of two-, three-, and four-electron atomic ions," Naturwissenschaften, vol. 97, no. 7, pp. 689-696, 2010.

[4] P. A. M. Dirac, Principles of Quantum Mechanics, Oxford University Press, Oxford, UK, 1930.

[5] W. E. Lamb and R. C. Retherford, "Fine structure of the hydrogen atom by a microwave method," Physical Review, vol. 72, no. 3, pp. 241-243, 1947.

[6] W. E. Lamb and R. C. Retherford, "Fine structure of the hydrogen atom. Part I," Physical Review, vol. 79, no. 4, pp. 549-572, 1950.

[7] W. E. Lamb and R. C. Retherford, "Fine structure of the hydrogen atom. Part II," Physical Review, vol. 81, no. 2, pp. 222-232, 1951.
[8] J. D. Garcia and J. E. Mack, "Energy level and line tables for one-electron atomic spectra," Journal of the Optical Society of America, vol. 55, no. 6, pp. 654-676, 1965.

[9] J. Midtdal and K. Aashamar, "Perturbation theory expansions through 20th order of mass polarization correction, relativistic effects and Lamb shift of the two-electron system," Physica Norvegica, vol. 2, pp. 99-108, 1967.

[10] C. E. Moore, Ionization Potentials and Ionization Limits Derived from the Analysis of Optical Spectra. NSRDS-NBS 34, US Department of Commerce, Washington, DC, USA, 1970.

[11] C. E. Moore, Atomic Energy Levels, vol. 1, US Department of Commerce, Washington, DC, USA, 1949.

[12] C. E. Moore, Atomic Energy Levels, vol. 2, US Department of Commerce, Washington, DC, USA, 1952.

[13] C. E. Moore, Atomic Energy Levels, vol. 3, US Department of Commerce, Washington, DC, USA, 1958.

[14] W. M. Haynes, Ed., CRC Handbook of Chemistry and Physics, CRC Press, Boca Raton, Fla, USA, 92nd edition, 2011-2012.

[15] P. F. Lang and B. C. Smith, "Relativistic corrections for calculating ionization energies of one to five electron isoelectronic atomic ions," ISRN Inorganic Chemistry, vol. 2013, Article ID 689040, 10 pages, 2013.

[16] A. Einstein, The Meaning of Relativity, Chapman and Hall, London, UK, 1967.

[17] G. W. Drake, "Theoretical energies for the $n=1$ and 2 states of the helium isoelectronic sequence up to $Z=100$," Canadian Journal of Physics, vol. 66, no. 7, pp. 586-611, 1988.

[18] E. U. Condon and H. Odabasi, Atomic Struture, Council of Urban Professionals, New York, NY, USA, 1980.

[19] I. R. Williams and M. W. Williams, Basic Nuclear Physics, Newnes, London, UK, 1962.

[20] N. Agmon, "Lonization potentials for isoelectronic series," Journal of Chemical Education, vol. 65, no. 1, pp. 42-44, 1988.

[21] J. C. Slater, "Atomic shielding constants," Physical Review, vol. 36, no. 1, pp. 57-64, 1930.

[22] P. W. Atkins, Molecular Quantum Mechanics Part III, Oxford University Press, Oxford, UK, 1970.

[23] P. F. Lang and B. C. Smith, "Ionization energies of atoms and atomic ions," Journal of Chemical Education, vol. 80, no. 8, pp. 938-946, 2003.

[24] P. F. Lang and B. C. Smith, "Ionization energies of lanthanides," Journal of Chemical Education, vol. 87, no. 8, pp. 875-881, 2010.

[25] G. Wulfsberg, Inorganic Chemistry, University Science Books, Sausalito, Calif, USA, 2000.

[26] C. A. Couson, Valence, Oxford University Press, London, UK, 2nd edition, 1961. 

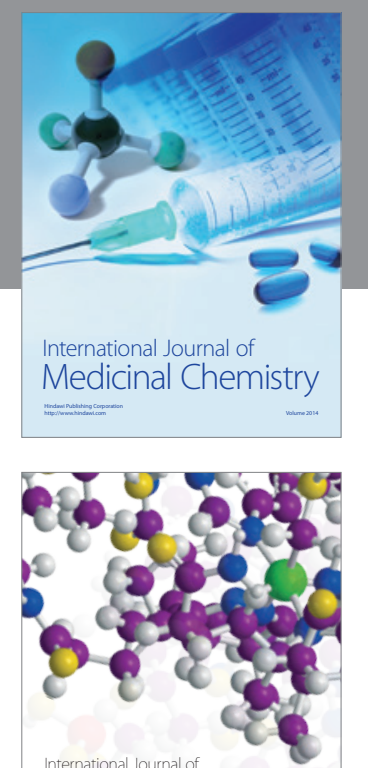

\section{Carbohydrate} Chemistry

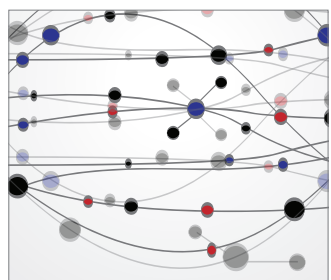

The Scientific World Journal
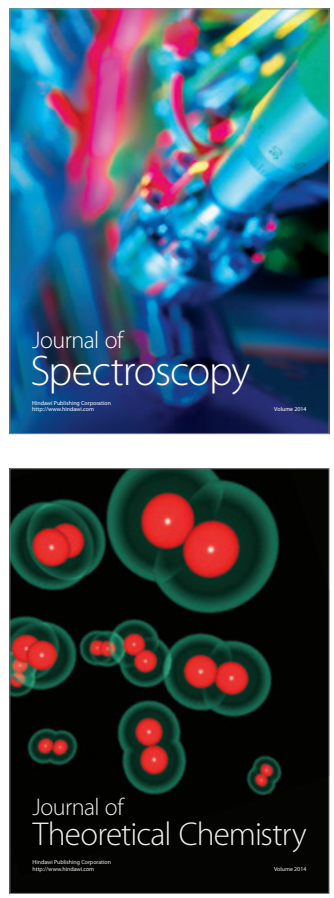
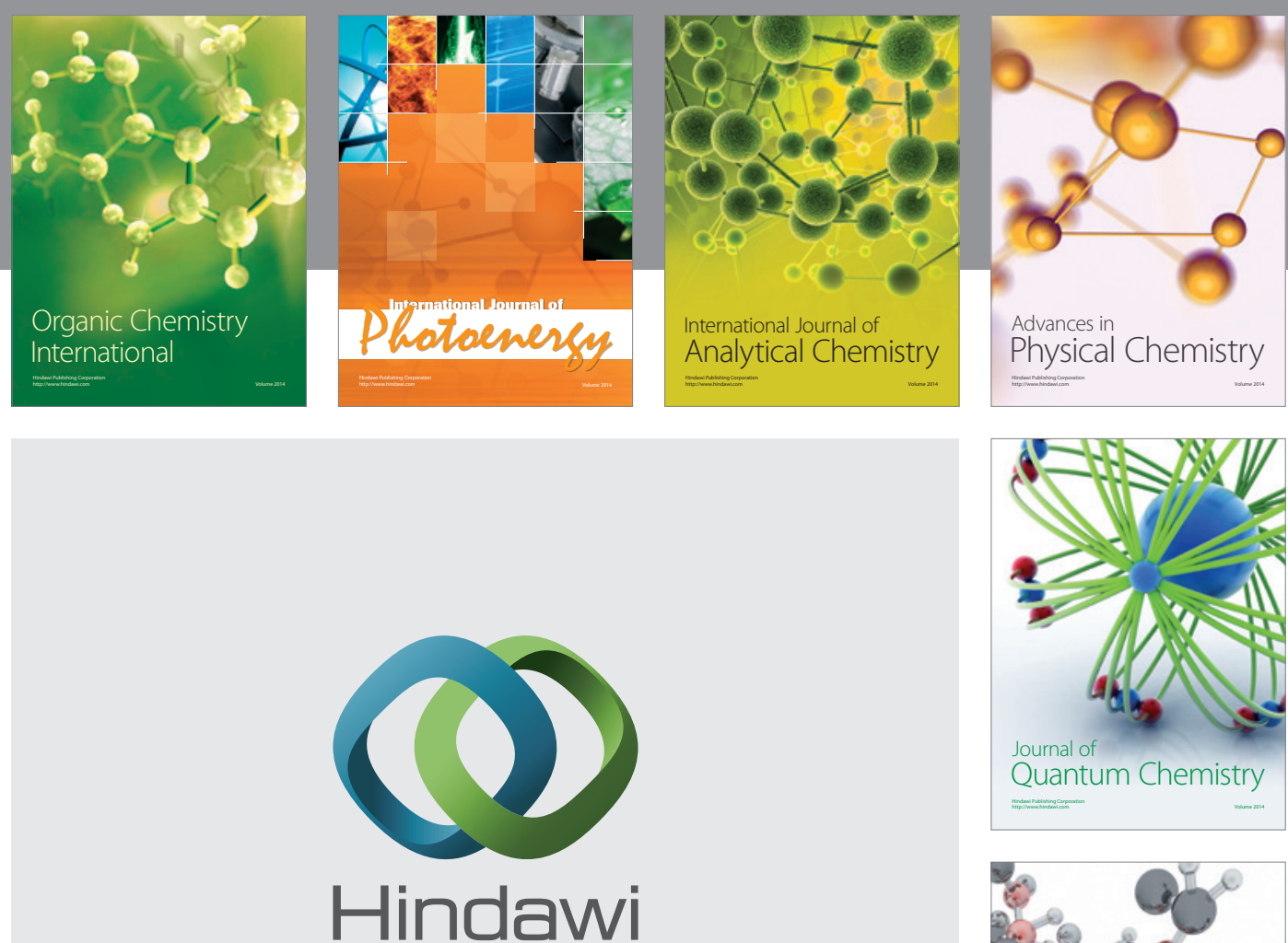

Submit your manuscripts at

http://www.hindawi.com

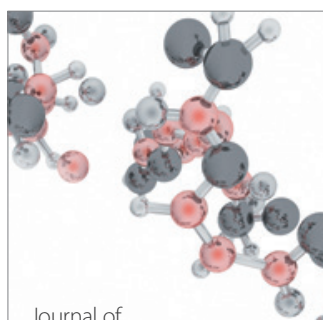

Analytical Methods

in Chemistry

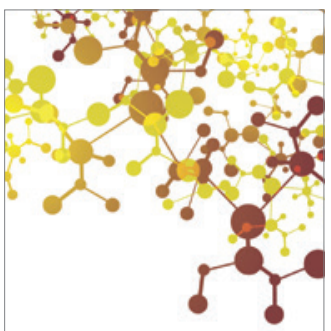

Journal of

Applied Chemistry

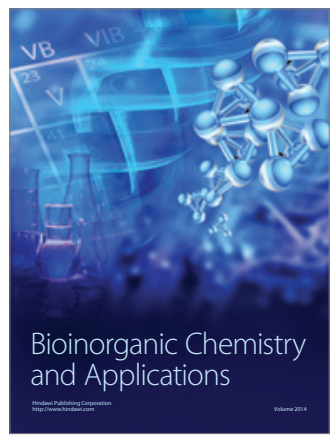

Inorganic Chemistry
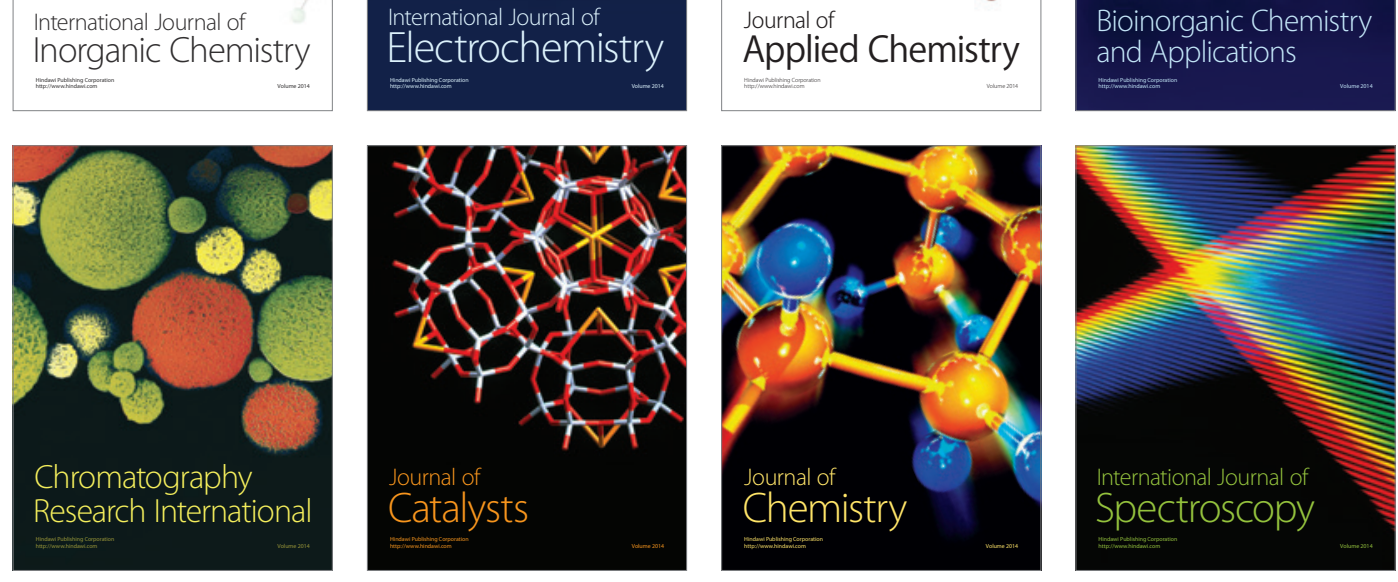